\title{
Description of composite bosons in discrete models
}

\author{
Paula Céspedes, ${ }^{1}$ Elena Rufeil-Fiori, ${ }^{2}$ P. Alexander Bouvrie, ${ }^{3}$ Ana P. Majtey, ${ }^{2}$ and Cecilia Cormick ${ }^{2}$ \\ ${ }^{1}$ FAMAF, Universidad Nacional de Córdoba, Ciudad Universitaria, X5016LAE, Córdoba, Argentina \\ ${ }^{2}$ Instituto de Física Enrique Gaviola, CONICET and Universidad Nacional de Córdoba, Ciudad Universitaria, \\ X5016LAE, Córdoba, Argentina \\ ${ }^{3}$ Centro Brasileiro de Pesquisas Físicas, Rua Dr. Xavier Sigaud 150, 22290-180 Rio de Janeiro, Rio de Janeiro, Brazil
}

(Received 18 December 2018; revised manuscript received 15 May 2019; published 8 July 2019)

\begin{abstract}
The understanding of the behavior of systems of identical composite bosons has progressed significantly in connection with the analysis of the entanglement between constituents and the development of coboson theory. The basis of these treatments is a coboson ansatz for the ground state of a system of $N$ pairs, stating that in appropriate limits this state is well approximated by the account of Pauli exclusion in what would otherwise be the product state of $N$ independent pairs, each described by the single-pair ground state. In this work we study the validity of this ansatz for particularly simple problems, and show that short-range attractive interactions in very dilute limits and a single-pair ground state with very large entanglement are not enough to render the ansatz valid. On the contrary, we find that the dimensionality of the problem plays a crucial role in the behavior of the many-body ground state.
\end{abstract}

DOI: 10.1103/PhysRevA.100.012309

\section{INTRODUCTION}

The fact that composite systems made up of an even number of fermionic constituents behave in practice like elementary bosons is long known [1-3], but a rigorous understanding of this behavior has only been gained recently. Such advancement has taken place including elements of quantum information theory, in particular entanglement theory [4,5], but has been especially accomplished by the development of coboson theory [6-8]. This formalism, originally designed to study phenomena such as excitations in a crystalline solid by means of excitons or superconductivity through Cooper pairs, dealing with pairs of fermions as compound bosons [8], was also successfully applied to a larger variety of systems [9], including molecular Bose-Einstein condensates (BECs) in ultracold interacting Fermi gases [10-13]. Manifestations of the effects of Pauli exclusion in composite bosons made of fermions have been analyzed for thought interference experiments in Refs. [14,15] and for potential implementations with condensates in Ref. [16]. We note, however, that although the coboson treatment provides a good approach for the description of Feshbach molecules [10-12], its application for the understanding of atomic Cooper pairs is nontrivial [17], and it remains unclear whether the description of the BEC-BCS crossover in terms of coboson theory is possible.

Precisely because of the relevance of coboson theory and its success in describing several physical phenomena, an understanding of its regime of validity is especially desirable. In particular, it is not evident when a key element of the theory, namely, the so-called coboson ansatz for the ground state, provides an appropriate description of the zero-temperature state of a system of $N$ pairs. The ansatz approximates the ground state of $N$ composite bosons by a state which is given by the repeated action over the vacuum of an operator creating one pair in the single-pair ground state, including a proper additional normalization to account for the effect of Pauli exclusion. Loosely speaking, one expects this ansatz to be valid when the constituent interactions are sufficiently short ranged, the system is sufficiently dilute, and the ground state for a single pair is highly entangled [8,11].

In this work we show in a particularly simple example that these conditions are not sufficient for the ansatz to be valid, and that the dimensionality of the problem actually plays a key role. This is due to the fact that one-dimensional models, even with short-range interactions, can lead to longrange correlations in the ground states of several pairs. It is important to stress that coboson theory was not developed for one-dimensional (1D) problems, and that our observations do not undermine the importance of the theory. On the contrary, we expect to contribute to the usefulness of this theoretical body of work by helping to establish more clearly its limits of applicability.

In particular, the model we consider was motivated by the one introduced in Ref. [14], where the coboson ansatz was taken as the initial state to study the effects of compositeness in a thought interference experiment. Although the use of the coboson ansatz in Ref. [14] is not at all essential for the analysis presented, the article can convey the mistaken impression that the ansatz is valid for the system considered, namely, a 1D chain of discrete sites along which the constituent fermions can hop, and including a short-range interaction between fermions of different species such that pairs are always strongly bound.

Here, we analyze this model in the situation where the coboson ansatz is expected to work best, namely, for maximum entanglement between constituents, and focusing on the simplest case of two pairs. We show that as the particle density becomes lower, the coboson state does not approach the true ground state of the system, and indeed the fidelity decreases, reaching a limiting value of $8 / \pi^{2}$. We also show 
that extending this model to a "ladder" with a fixed width of $n$ sites, to allow pairs to cross each other, does not significantly modify this result. The reason for this behavior is the long-range character of the correlations appearing for one-dimensional settings, which cannot be captured by the ansatz. On the contrary, when the system is made truly two dimensional, the coboson ansatz becomes a good description of the system as long as it is dilute enough.

This article is organized as follows: In Sec. II we introduce briefly some very basic elements of the coboson formalism. In Sec. III we give the details of the model under consideration, the single-pair ground manifold and the effective Hamiltonian for the ground manifold of $N$ pairs. In Sec. IV we give the exact solution for the ground state of two pairs and compare it with the coboson ansatz, observing that the fidelity with the true ground state decreases as the number of sites is increased. Section V presents an analysis of the two-dimensional generalization of the system, showing that the coboson ansatz behaves well in this case, and in Sec. VI we provide a discussion of the results and summarize our conclusions. Finally, in the Appendix we include a short study of the behavior of the energy of two pairs for the two-dimensional case.

\section{THE COBOSON GROUND STATE}

We consider a system of identical composite bosons, each made of two distinguishable fermions. This section provides a brief overview of the coboson ansatz for the ground state of $N$ such pairs. For a more complete introduction to coboson theory, we refer the reader to Refs. [4-6,8,18]. For a given Hamiltonian corresponding to a single pair, the ground state $|\psi\rangle$ defines the coboson creation operator $c^{\dagger}$, namely, the operator which acts on the vacuum creating a single pair in the ground state, $|\psi\rangle=c^{\dagger}|0\rangle$. In the Schmidt basis, this operator can be written as [4]

$$
c^{\dagger}=\sum_{\alpha=1}^{S} \sqrt{\lambda_{\alpha}} a_{\alpha}^{\dagger} b_{\alpha}^{\dagger}
$$

with

$$
\lambda_{1} \geqslant \lambda_{2} \geqslant \cdots \geqslant 0
$$

the Schmidt coefficients satisfying

$$
\sum_{\alpha=1}^{S} \lambda_{\alpha}=1
$$

and $S$ the (finite or infinite) Schmidt rank. The operators $a_{\alpha}^{\dagger}$ and $b_{\alpha}^{\dagger}$ create one particle of kind $a$ or $b$, respectively, in the corresponding Schmidt modes $\alpha$. The operator $c$ obeys the following commutation relations:

$$
[c, c]=\left[c^{\dagger}, c^{\dagger}\right]=0, \quad\left[c, c^{\dagger}\right]=1-\Delta,
$$

where

$$
\Delta=\sum_{\alpha=1}^{S} \lambda_{\alpha}\left(a_{\alpha}^{\dagger} a_{\alpha}+b_{\alpha}^{\dagger} b_{\alpha}\right)
$$

One can write a normalized state of $N$ composite bosons obtained after acting $N$ times with the coboson creation operator in the form $[4,6]$

$$
|N\rangle=\frac{\left(c^{\dagger}\right)^{N}}{\sqrt{N ! \chi_{N}}}|0\rangle,
$$

where $\chi_{N}$ is the compositeness normalization factor $[4,6,18,19]$ which depends on the Schmidt coefficients and which accounts for the subnormalization of the state resulting in adding $N$ bifermions to the vacuum. For composite bosons made of two fermions the normalization factor takes the form [4]

$$
\chi_{N}=N ! \sum_{p_{N}>p_{N-1}>\cdots>p_{1}}^{S} \lambda_{p_{1}} \lambda_{p_{2}} \cdots \lambda_{p_{N}},
$$

which is the elementary symmetric polynomial [20]. The idea that the state $|N\rangle$ provides a good approximate description of the ground state of a system of $N$ cobosons is a key element of coboson theory, and we refer to this in the following as the coboson ansatz.

For the case $N=2$, the normalization coefficient is equal to $\chi_{2}=1-P$, with $P=\sum_{\alpha} \lambda_{\alpha}^{2} \leqslant 1$ the purity of the reduced density matrix of one of the constituent particles of a pair in the ground state $|\psi\rangle$. In general, the behavior of pairs as approximate elementary bosons can be related with the normalization coefficients, and bosonic behavior is recovered when $\chi_{N} / \chi_{N-1} \simeq 1[4,19,21,22]$.

\section{THE MODEL}

The problem we consider is a one-dimensional array of $L$ sites with two species of fermions that can hop along them. Fermions of different species have a very strong attraction, so that if the numbers of particles of both species are equal then the low-energy manifold has all particles in pairs. More precisely, the Hamiltonian takes the form

$$
\begin{aligned}
H= & -U_{0} \sum_{j=1}^{L} a_{j}^{\dagger} a_{j} b_{j}^{\dagger} b_{j} \\
& +\frac{J}{2} \sum_{j=1}^{L}\left(a_{j}^{\dagger} a_{j+1}+b_{j}^{\dagger} b_{j+1}+\text { H.c. }\right),
\end{aligned}
$$

where $a_{j}\left(a_{j}^{\dagger}\right)$ destroys (creates) a particle of type $a$ in site $j$, $b_{j}\left(b_{j}^{\dagger}\right)$ does the same for a particle of type $b$, and we assume for definiteness that the operators associated with particles of different kinds commute (the results are the same if they anticommute [8]).

We consider that the interaction energy is much stronger than the hopping, i.e., $U_{0} \gg J$. We then study this problem analytically using perturbation theory. As shown in the following, the restriction to the limit when particles always tunnel in pairs makes our system an instance of the hard-core Bose-Hubbard model, which is equivalent to the Heisenberg model [23-26]. We note that this limit of very strongly bound pairs is the one studied in Ref. [14], and it is also particularly relevant for our interests since it is the situation where the coboson description should be most appropriate. We remark that the problem considered in Ref. [14] includes also sitedependent energies, as a free parameter to control the amount 
of entanglement in the single-pair ground state. Here for simplicity we focus on the case where the coboson ansatz is supposed to work best, namely, when the entanglement is maximum. This corresponds to the translation-invariant case with all site energies equal and periodic boundary conditions. In the following we derive the effective Hamiltonian for the lowest-energy manifold in this model, for a single pair and for $N$ pairs.

\section{A. Single-pair basis: Ground manifold}

The Hilbert space of a single pair of particles, one of each kind, divides into a ground manifold composed by the states where the particles are paired (i.e., occupying the same site), containing $L$ states, and an excited manifold where the particles are not paired, with dimension $L^{2}-L$. The ground manifold energy, to zeroth order in the hopping, is $-U_{0}$, while the excited manifold has zero energy up to the same order. Using perturbation theory, we can find the approximate eigenstates within each of these highly degenerate manifolds.

As already explained in Ref. [14], to first order the hopping Hamiltonian for the ground manifold vanishes, so that the first nonzero correction is of second order and has the form

$$
H_{g} \simeq-U_{0}-P_{g} \frac{H_{J}^{2}}{U_{0}} P_{g},
$$

where $P_{g}$ is the projector onto the ground manifold, and $H_{J}$ is the hopping part of the Hamiltonian. It is straightforward to see that this gives (for the case of a single pair)

$$
H_{g}^{(1)} \simeq-U_{0}-J^{\mathrm{eff}}-\frac{J^{\mathrm{eff}}}{2} \sum_{j=1}^{L}(|j, j\rangle\langle j+1, j+1|+\text { H.c. })
$$

so that particles always tunnel in pairs. Here, the effective tunneling constant is given by

$$
J^{\mathrm{eff}}=\frac{J^{2}}{U_{0}} .
$$

We note that the effective Hamiltonian is independent of the sign of $J$; indeed, a change of sign in $J$ in the original Hamiltonian (8) can be reabsorbed by a sign flip in the creation and annihilation operators corresponding to all odd (or all even) sites, and this sign flip becomes irrelevant when only pairs can tunnel.

It is also easy to diagonalize this Hamiltonian with a Fourier transformation. The coboson operators which create the single-pair eigenstates within this manifold are thus found to be of the form

$$
c_{k}^{\dagger}=\frac{1}{\sqrt{L}} \sum_{j} e^{-2 \pi i k j / L} a_{j}^{\dagger} b_{j}^{\dagger}
$$

with corresponding energies

$$
E_{k}=-U_{0}-2 J^{\mathrm{eff}} \cos ^{2}(k \pi / L),
$$

where $k$ runs from 0 to $L-1$. Except for the lowest state within this manifold (and for even $L$ also the highest), the eigenstates are doubly degenerate.

The single-pair ground-state energy is thus

$$
E_{0}=-U_{0}-2 J^{\mathrm{eff}} \text {, }
$$

and the ground state is

$$
|G\rangle_{N=1}=\frac{1}{\sqrt{L}} \sum_{j}|j, j\rangle
$$

with the ground-state coboson creation operator given by $c^{\dagger}=c_{0}^{\dagger}$. This greatly simplifies some calculations, because all Schmidt coefficients of this state are equal to $1 / L$, and it is straightforward to compute the form of the coboson ansatz for the ground state of $N$ pairs and the corresponding normalization factors (see Sec. IV). We note also that in this case $S=L$, and the entanglement between the components of a single pair in the ground state is characterized by the purity $P=1 / L$, corresponding to a maximally entangled state for each fixed number of sites.

\section{B. Effective Hamiltonian for the ground manifold of $N$ pairs}

Following similar lines as before, one can use perturbation theory for the effective Hamiltonian of the ground manifold for the case of several pairs. Once more, it is trivial to see that the ground manifold is formed by the states in which all particles are paired, and to zeroth order in the hopping the energy of this manifold is $-N U_{0}$. In the following we analyze the corrections when second-order terms in the hopping are introduced.

In an analogous manner as in the single-pair case, the effective Hamiltonian for the ground manifold takes the form

$$
H_{g} \simeq-N U_{0}-P_{g} \frac{H_{J}^{2}}{U_{0}} P_{g} .
$$

Now the projection to the ground manifold of the term proportional to $H_{J}^{2}$ has an additional term coming from the fact that hopping of a particle out of a given site might be forbidden if there is already a particle sitting there. This leads to the form

$$
\begin{gathered}
H_{g}^{(N)} \simeq-N\left(U_{0}+J^{\mathrm{eff}}\right)+J^{\mathrm{eff}} \sum_{j} N_{j} N_{j+1} \\
-\frac{J^{\mathrm{eff}}}{2} \sum_{j}\left(T_{j}^{+}+T_{j}^{-}\right),
\end{gathered}
$$

where $N_{j}$ is the number of pairs in site $j$, and $T_{j}^{ \pm}$are the operators that correspond to hopping of a pair from site $j$ to $j \pm 1$. For the case $N=1$ this clearly reduces to the singlepair effective Hamiltonian of the ground manifold given by Eq. (10).

One can see from the form of the ground-manifold Hamiltonian for $N$ pairs that, as in the single-pair case, there is a hopping term that will tend to delocalize the cobosons, but now there is an additional interaction between sites that will compete with the hopping. This interaction is repulsive, and therefore one expects that the ground state will have delocalized pairs but which are unlikely to be found next to each other. The exact ground state of the effective Hamiltonian and its energy for the case of two pairs are discussed in Sec. IV.

\section{Relation with the Heisenberg model}

Discrete hard-core boson models are equivalent to spin-1/2 systems, and indeed Hamiltonian (17) is equivalent to the 
Heisenberg Hamiltonian for a chain of spins $1 / 2$, by means of the identifications $a_{j} b_{j} \equiv \sigma_{j}^{-}$, the spin lowering operator, $N_{j} \equiv\left(\sigma_{j}^{z}+1\right) / 2$. Tunneling terms of the form $T_{j}^{+}+T_{j}^{-}$can then be written as interactions of the form $\sigma_{j}^{x} \sigma_{j+1}^{x}+\sigma_{j}^{y} \sigma_{j+1}^{y}$, while the term of the form $N_{j} N_{j+1}$ corresponds to an interaction through $\sigma_{z}$ plus a global field along the $z$ direction. In order to obtain the Heisenberg Hamiltonian one has to additionally apply on every other spin a rotation about the $z$ axis in order to flip the sign of the corresponding $\sigma_{x}$ and $\sigma_{y}$ operators. This only works for an even number of sites, but for big systems we do not expect the parity of the number of sites to play a crucial role. form

The effective ground-manifold Hamiltonian then takes the

$$
H_{g}^{(N)} \equiv-N U_{0}+\frac{J^{\mathrm{eff}}}{4}\left(H_{H}-L\right),
$$

where $H_{H}$ is the Heisenberg Hamiltonian:

$$
H_{H}=\sum_{j} \vec{\sigma}_{j} \cdot \vec{\sigma}_{j+1}
$$

The ground-state energy for the system of $N$ pairs can be obtained from the minimum energy of the Heisenberg Hamiltonian in the manifold corresponding to $N$ particles, which fixes the total projection of the spin along $z, \sigma_{T}^{z}=2 N-L$.

This means the problem can be approached with the Bethe ansatz [25], and its properties have been studied extensively [26]. In general, it is not possible to find an exact analytical solution of the Heisenberg model for arbitrary values of $N$. However, for the particular case with two particles only, an exact solution can be written for the ground state, as will be discussed in Sec. IV.

\section{EXACT GROUND STATE FOR TWO PAIRS, AND COMPARISON WITH THE COBOSON ANSATZ}

For the case of two pairs, the exact analytical solution of the effective Hamiltonian is known and has the form [25]

$$
|G\rangle_{N=2}=A \sum_{j_{1}<j_{2}} \sin \left[\pi \frac{d\left(j_{1}, j_{2}\right)-1 / 2}{L-1}\right]\left|j_{1}, j_{2}\right\rangle,
$$

where $A$ is a normalization factor and $d\left(j_{1}, j_{2}\right)$ is the distance between the two occupied sites, taken mod $L$. It is convenient to notice that although the mapping to the Heisenberg Hamiltonian as described above was valid for even $L$ only, this expression for the ground state holds also for $L$ odd.

The coboson ansatz for the ground state of a system of many particles, when the numbers of particles of each kind are both equal to $N$, is given by Eq. (6). Given the form of the operator $c_{0}^{\dagger}$ in Eq. (12), the ansatz for this problem leads to

$$
|N\rangle=\left[\left(\begin{array}{c}
L \\
N
\end{array}\right)\right]^{-1 / 2} \sum_{j_{1}<j_{2}<\cdots<j_{N}} a_{j_{1}}^{\dagger} b_{j_{1}}^{\dagger} \cdots a_{j_{N}}^{\dagger} b_{j_{N}}^{\dagger}|0\rangle .
$$

It is straightforward to notice that this ansatz for the ground state cannot capture at all the effects of the effective repulsion appearing in the Hamiltonian for the ground manifold of the $N$ particles. Indeed, it is entirely determined by the hopping term, since this is the only term in the single-pair Hamiltonian. Nevertheless, one could expect that this is still a good

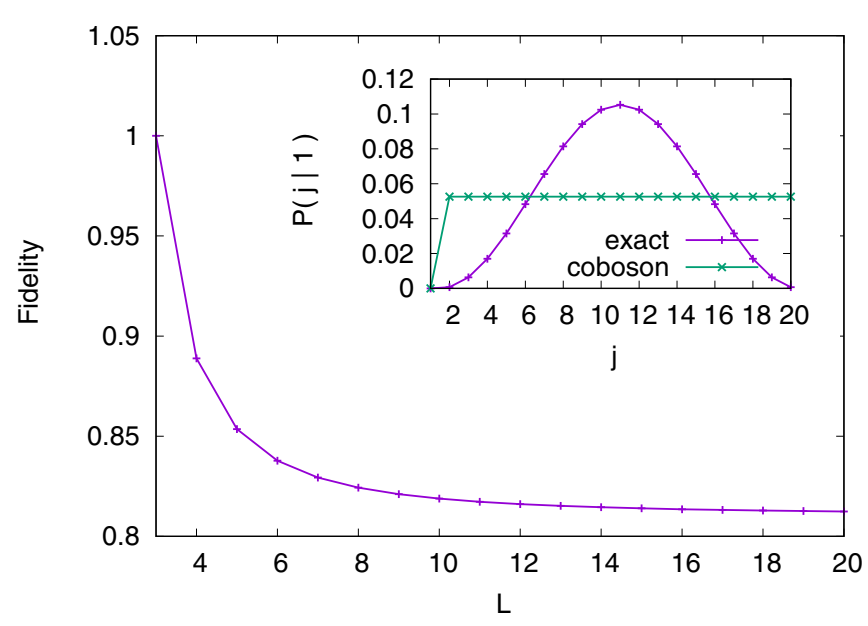

FIG. 1. Fidelity between the analytical ground state and the coboson ansatz for two pairs in the very strongly bound limit, in a onedimensional lattice with $L$ sites and periodic boundary conditions. Inset: Probability to find a pair in each site of the lattice conditional on having found one pair in the first site, for $L=20$, evaluated over the analytical ground state (20) and over the coboson ansatz, displayed with violet + and turquoise $\times$ symbols, respectively. All depicted quantities are dimensionless.

approximation in the limit of low densities, for which coboson theory was developed $[8,11,12]$. In that limit, two cobosons are anyway very unlikely to be found next to each other, so corrections due to repulsion may be negligible. However, this turns out not to be the case.

For the particular case $N=2$, which is the first nontrivial scenario to which coboson theory can be applied and for which the analytical solution of the problem is given in Eq. (20), one can calculate the fidelity between the exact ground state and the coboson ansatz. This is defined as

$$
\mathcal{F}\left(|N\rangle,|G\rangle_{N}\right)=\left|\langle N \mid G\rangle_{N}\right|^{2}
$$

and one finds that $\mathcal{F}$ actually decreases with the number of sites. The general calculation is cumbersome, but the limit $L \rightarrow \infty$ is particularly simple because a continuum limit can be taken turning sums over sites into integrals. For an infinite number of sites, i.e., for pair density tending to zero, the fidelity approaches the value $\mathcal{F}_{\infty}=8 / \pi^{2} \simeq 0.81$. The behavior of the fidelity as a function of $L$ is shown in Fig. 1. The high symmetry of our model, resulting from both the hard-core character of the bosons and the translational invariance, leads to a fidelity equal to 1 in a lattice with $L=N+1$ for an arbitrary number of pairs, $N$. It is worth noting that even though for this particular model the coboson ansatz works well for small values of $L$ corresponding to a high-density regime, the ansatz was formulated to work in very dilute regimes, namely, $L \gg N[8,11,12]$. As we are interested in analyzing the validity of the ansatz we focus only on the situation for which the coboson formalism has been developed.

Numerical calculations show that the fidelity between the coboson ansatz and the ground state obtained numerically also decreases with the number of sites for larger values of particles. This is reasonable since the coboson ansatz is not 
expected to improve as the number of particles gets larger [27]; indeed, in coboson theory the dominant terms in an expansion in powers of the particle density are determined by the solutions of the problems of one and two pairs $[7,10]$. This is why we restrict our report to the most significant case of two pairs.

We remark that it is possible to compute analytically the energy associated with the coboson ansatz for the ground state, and its value does converge to the right ground energy as the number of sites increases. Indeed, the ground-state energy of the effective Hamiltonian for two pairs is equal to

$$
E_{G, N=2}=-2 U_{0}-4 J^{\mathrm{eff}} \cos ^{2}\left(\frac{\pi}{2(L-1)}\right),
$$

whereas the coboson ansatz leads to the result

$$
E_{G, N=2} \simeq\left\langle 2\left|H_{g}^{(2)}\right| 2\right\rangle=-2 U_{0}-4 J^{\mathrm{eff}}+4 J^{\mathrm{eff}} \frac{1}{L-1} .
$$

Thus, the two expressions approach each other as $L$ tends to infinity. However, this is merely due to the fact that the contribution of the interactions to the energy goes to zero as the pair density becomes negligible. Indeed, for large $L$ the ground-state energy of two pairs tends to twice the value of the single-pair ground-state energy, Eq. (14), as one would expect.

The reason for the bad performance of the coboson ansatz can be traced back to the long-range character of the correlations between pairs in the true ground state (20). Given the translational invariance of the model and the fact that site occupation is at most equal to 1 , the expectation value $\left\langle N_{1} N_{j}\right\rangle$ can be related to the conditional probability $P(j \mid 1)$ of finding one pair in position $j$ given that another pair was found located at site 1 , as

$$
P(j \mid 1)=\frac{\left\langle N_{1} N_{j}\right\rangle}{\left\langle N_{1}\right\rangle}=L\left\langle N_{1} N_{j}\right\rangle=L\left|\langle 1, j \mid G\rangle_{2}\right|^{2} .
$$

For the exact ground state (20), the probability $P(j \mid 1)$ is proportional to $\sin ^{2}[\pi(j-3 / 2) /(L-1)]$; i.e., it varies smoothly from zero for short distances $(j \simeq 1)$ to the maximum value when the pairs are at opposite positions in the chain $(j \simeq$ $L / 2$ ). The coboson ansatz, on the contrary, predicts a flat probability distribution with equal probabilities for all nonzero distances between pairs, i.e., $P(j \mid 1)=1 /(L-1) \forall j \neq 1$. The contrast between the spatial correlations present in these two states is illustrated in the inset of Fig. 1, which shows the conditional probability $P(j \mid 1)$.

\section{THE TWO-DIMENSIONAL CASE}

The failure of coboson theory to give a good approximate description of the ground state in the 1D toy model studied is nontrivial, since the interactions are very short ranged, pairs are strongly bound, and the single-pair ground state can contain arbitrarily high entanglement. The reason why coboson theory is not applicable in this model seems to be that even for very low densities the ground state of two bounded pairs presents infinite-range correlations between the pairs. But this, in general, cannot be known until one solves for the ground state, which is exactly what one wishes to avoid by using the coboson ansatz. This naturally leads to the question, is there a key feature of the model that allows one to identify when coboson theory starts failing? Some rapid conjectures come to mind: the failure can be due to the 1D character of the model, the impenetrability of pairs (which can never cross each other), or the discretization of space. In this section we analyze some of these possibilities.

We thus consider the simplest extension of the previous model: another lattice with $n \times L$ sites, so pairs can go around each other. For definiteness, we take periodic boundary conditions in both directions (i.e., a torus geometry). The basis of states of one fermion of either kind, or of one composite boson in the strongly bound regime, is given by the set of possible positions $|j\rangle=\left|\left(j_{x}, j_{y}\right)\right\rangle$ with $j_{x}=1, \ldots, L$ and $j_{y}=$ $1, \ldots, n$. The Hamiltonian is analogous to the one in Eq. (17), except that interactions and tunneling can involve any pair of neighboring sites. Without breaking the translational invariance that is key for the simple form of the coboson ansatz and for the maximum entanglement between pair components, one can take two different effective tunneling constants, one for each direction, i.e., $J_{v}^{\text {eff }}=J_{v}^{2} / U_{0}$ with $v=x, y$. This leads to the Hamiltonian

$$
\begin{aligned}
H_{g}^{(N)} \simeq & -N\left(U_{0}+J_{x}^{\mathrm{eff}}+J_{y}^{\mathrm{eff}}\right) \\
& +\sum_{\nu=x, y} J_{v}^{\mathrm{eff}}\left[\sum_{\langle i, j\rangle_{v}} N_{i} N_{j}-\frac{1}{2} \sum_{j}\left(T_{j}^{\nu+}+T_{j}^{\nu-}\right)\right],
\end{aligned}
$$

where $\langle i, j\rangle_{v}$ denotes pairs of sites that are neighbors along direction $v$, and $T_{j}^{\nu \pm}$ are the operators that correspond to hopping of a pair from site $j=\left(j_{x}, j_{y}\right)$ along direction $v$ in the sense of increasing or decreasing $j_{v}$.

The ground state of a single pair in this model is again uniformly delocalized over all sites; thus, the coboson ansatz leads once more to a state with a very simple form which for two pairs in an $n \times L$ lattice reads

$$
|2\rangle=\frac{1}{2}\left[\left(\begin{array}{c}
L n \\
2
\end{array}\right)\right]^{-1 / 2} \sum_{j_{1} \neq j_{2}} a_{j_{1}}^{\dagger} b_{j_{1}}^{\dagger} a_{j_{2}}^{\dagger} b_{j_{2}}^{\dagger}|0\rangle,
$$

where $j_{k}=\left(j_{k, x}, j_{k, y}\right)$ for $k=1,2$. In order to obtain the ground state of two pairs numerically, we exploit the translational invariance restricting to the zero-momentum subspace. By doing this we are able to treat larger systems. The behaviors of the numerically obtained energy and the energy as given by the coboson ansatz for the ground state are shown in Figs. 6 and 7 (see the Appendix).

The results for the fidelity between the numerical ground state and the coboson ansatz are shown in Fig. 2, where for definiteness we take $J_{x}=J_{y}$. In each of the curves, we set $n$ fixed and decrease the pair density by increasing the value of $L$. Once more, the coboson ansatz fails to reproduce the features of the ground state in the limit of low densities. One can observe from the figure that, for each value of $n$, at first the fidelity increases with $L$, reaching a maximum value when $L \simeq n$. From that point, the fidelity decreases with $L$, and the system behaves one dimensionally. We have also analyzed cases where the tunneling constant is larger in one of the directions than in the other, and found that the values of the fidelity may vary with this choice but the decreasing trend of the fidelity for large $L$ is general. 


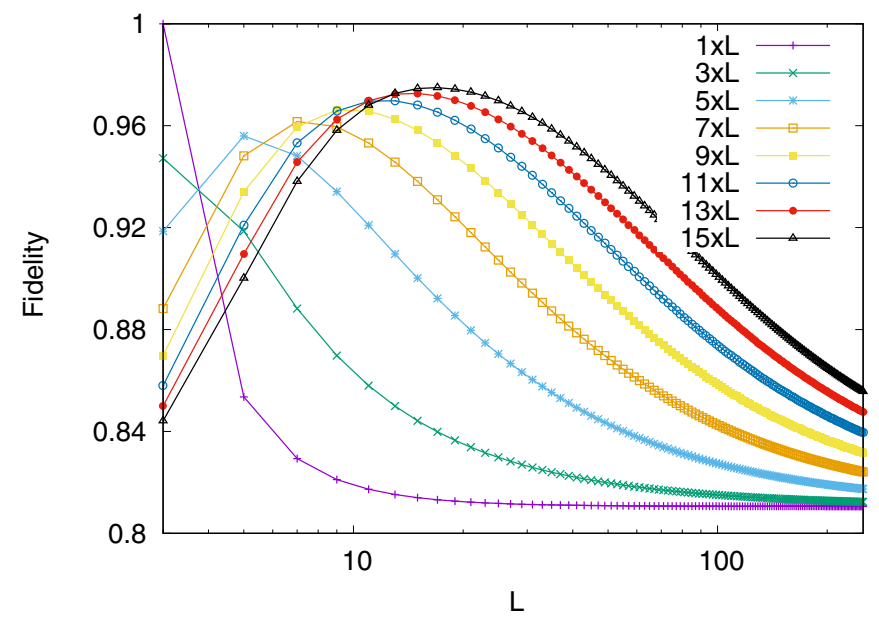

FIG. 2. Fidelity between the ground state found numerically and the coboson ansatz for two pairs in an $n \times L$ lattice with torus boundary conditions, and where the effective tunneling strength is the same in both directions. For fixed $n$, in all cases the fidelity decreases with $L$. All depicted quantities are dimensionless.

As in the previous section, the behavior of the fidelity can be understood in terms of the presence of long-range correlations in the positions of the pairs. Indeed, also for the $n \times L$ lattice one observes a pattern in the relative positions that resembles the $1 \times L$ case. As an illustration, Fig. 3 displays, for the case of equal tunnelings in both directions, the probability to find one pair relative to the position of the other for a $4 \times 18$ lattice, showing that the solution of this model also exhibits strong and long-range spatial correlations between the two pairs. On the other hand, the coboson ansatz (27) predicts

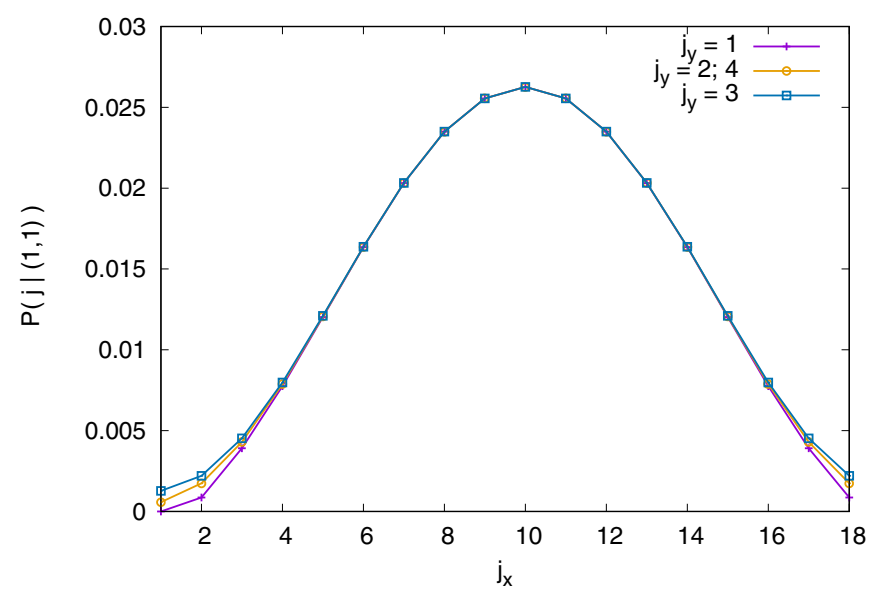

FIG. 3. Correlations between two pairs in the ground state of the $4 \times 18$ lattice with equal tunneling in both directions. Given the position of one pair at site $(1,1)$, the plot shows the probability to find the other pair as a function of the position in the same sublattice, $j_{y}=1$ (violet dashes) and in the other three sublattices $\left(j_{y}=2,3,4\right.$, where cases 2 and 4 are equal for symmetry reasons). Apart from the region which is closest to $(1,1)$, the probabilities for all four sublattices are very similar and resemble the sinusoidal distribution found for the $1 \times L$ lattice. All depicted quantities are dimensionless.

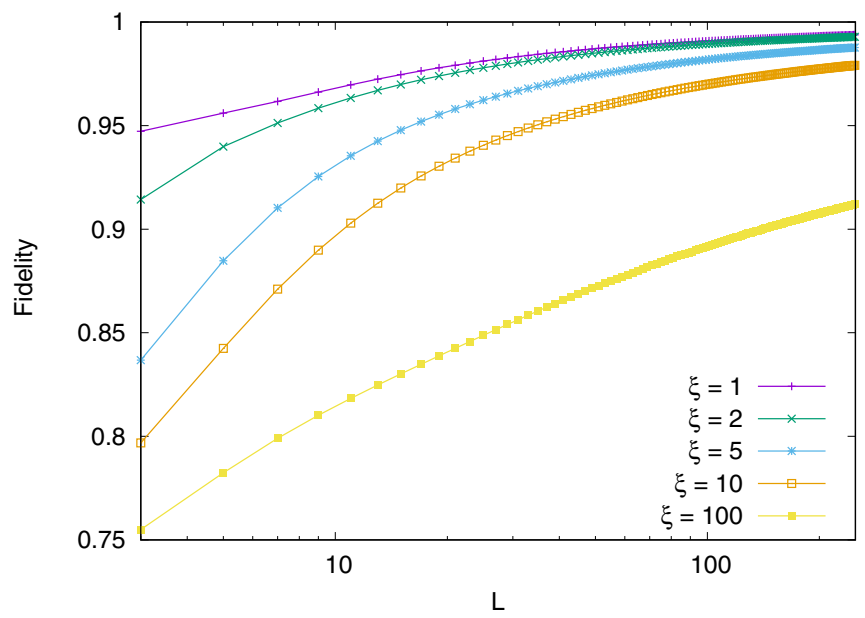

FIG. 4. Fidelity between the ground state found numerically and the coboson ansatz for an $L \times L$ torus, as a function of $L$. The anisotropies are, from top to bottom, given by $J_{x}^{\text {eff }} / J_{y}^{\text {eff }}=\xi$, for $\xi=1,2,5,10,100$. All depicted quantities are dimensionless.

a flat distribution with $P(j \mid(1,1))=1 /(L n-1)$ whenever $j \neq(1,1)$.

From Fig. 2 one can also observe that the maximum of the fidelity for each fixed $n$, found for $L \simeq n$, increases as a function of $n$. This suggests that the coboson ansatz is satisfactory in the truly two-dimensional case, i.e., when the low-density limit corresponds to a lattice size that increases in both dimensions. Figure 4 shows the results for the fidelity in an $L \times L$ lattice as a function of $L$, for several cases corresponding to equal or different tunneling strengths in each direction. As can be seen from the figure, in this case the description of the ground state in terms of the coboson ansatz improves as the number of sites is increased approaching the very dilute limit.

We note that all the cases plotted in Fig. 4 display a fidelity which increases with decreasing density, and the curves seem to asymptotically approach unit fidelity. However, the convergence is very slow and it strongly depends on the degree of anisotropy. This has a simple interpretation: for each finite value of $L$, if the tunneling in one of the directions is sufficiently large, there is a strong effective repulsion between pairs along that direction and each array of sites behaves effectively as a single cell. This makes the correlations in pair positions equivalent to the one-dimensional case. Nevertheless, fixing the values of the tunneling strengths and increasing sufficiently the value of $L$, the two-dimensional behavior is always recovered, with a characteristic correlation length along each direction that depends on the corresponding tunneling strength.

For comparison with the previous cases, Fig. 5 shows the correlations in the positions of the two pairs for a twodimensional lattice with $51 \times 51$ sites; more precisely, the probability distribution for the position of the second pair is displayed conditioned on the first pair being found in site $(1,1)$ and for the case when $J_{x}=J_{y}$. It can be seen that apart from a small region around the first site, the probability becomes relatively flat, which explains the good agreement with the coboson ansatz. These results indicate that the failure 


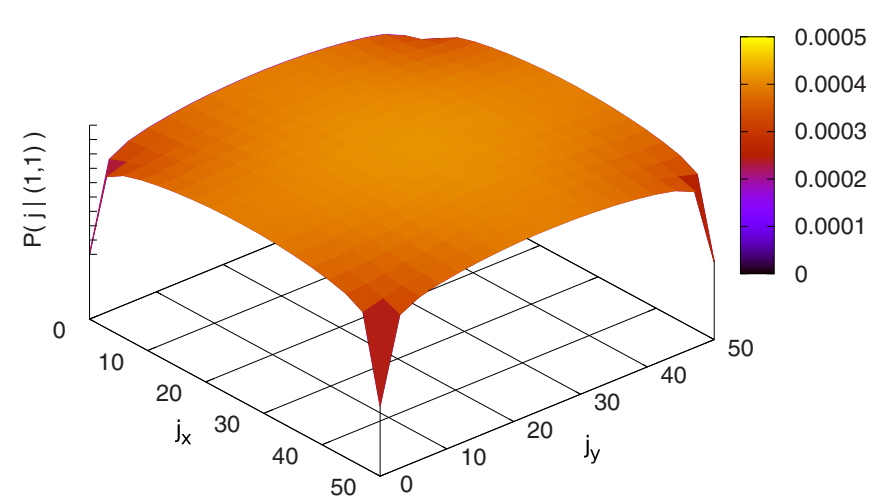

FIG. 5. Probability of finding a pair as a function of position given that there is a pair on site $(1,1)$, for an isotropic lattice with $51 \times 51$ sites. As one moves away from the occupied site the probability becomes relatively flat, allowing for a good description in terms of the coboson ansatz. All depicted quantities are dimensionless.

of the coboson ansatz observed in the previous section can be associated with the one-dimensional character of the model leading to long-range spatial correlations.

\section{CONCLUSIONS}

We have studied a toy model consisting of composite bosons strongly bound and tunneling along sites in a discrete lattice. Since we restrict to the case when the particles always tunnel in pairs, the model is equivalent to a hard-core BoseHubbard model, which is in turn equivalent to the Heisenberg model. The analysis of the one-dimensional case for two pairs has shown that the fidelity between the true ground state and the coboson ansatz decreases as the number of sites is increased. In the predictions of coboson theory for the case of $N$ pairs the dominant terms in an expansion with respect to pair density are given by the cases $N=1$ and $N=2[7,10]$. This means that whenever the coboson ansatz fails to provide an appropriate description of the system for two pairs, it will also fail for higher numbers. Since the translational symmetry of the model studied makes the ground-state entanglement between pair constituents maximum, we conjecture that the coboson ansatz cannot generally be expected to faithfully describe one-dimensional discrete models. We related this with the presence of long-range correlations, and verified that this failure is also found in a slightly more complex model with an $n \times L$ lattice, where the low-density limit is taken for fixed $n$ and increasing $L$. The fact that the fidelity between the true ground state and the coboson ansatz decreases with decreasing density was also observed for models where the tunneling constant was different in the two directions.

The same analysis was carried out for a two-dimensional model corresponding to an $L \times L$ lattice with full translational symmetry. The results for a system of two pairs in this case show that the fidelity between the numerically found ground state and the coboson ansatz improves as the density is decreased, and it seems to approach the ideal unit value as the number of sites approaches infinity. We note, however, that the fidelity is strongly dependent on the degree of anisotropy of the model. Indeed, in systems where the two tunneling constants are very different the coboson ansatz is markedly less satisfactory than in isotropic models with the same number of sites.

Our study reveals an aspect which is relevant for the fundamental understanding of when pairs of fermions are expected to behave approximately as elementary bosons, but which has received little attention so far. On top of a high amount of entanglement between constituents of a single pair, and a short-range character of the interactions so that for low densities pairs can be regarded as effectively independent, we observe a strong impact of the dimensionality of the system. One-dimensional models, namely, lattices where the number of sites in one dimension is much larger than in the other, tend to develop long-range correlations in the positions of the pairs which cannot be captured by the coboson ansatz. On the contrary, truly two-dimensional lattices display a behavior where the pairs can be approximately described as cobosons, with a fidelity that increases as the system becomes more dilute.

We note once more that our analysis restricts to the very strongly bound limit, such that the components of a pair always tunnel together and are always found in the same site. Systems of more loosely bound pairs are certainly of interest and can illustrate the gradual appearance of effective bosonic behavior. For instance, the one-dimensional extended Hubbard model, with a tunable nearest-neighbor interaction, has been the focus of Ref. [28]. Here, however, we consider only the limiting case of very bound pairs because it is the one where the conditions which are normally expected to render the coboson ansatz valid are best satisfied.

It is important also to stress for clarity that our findings are not directly connected with the well-known lack of condensation of a gas of noninteracting bosons at finite temperature and in the thermodynamic limit for less than three dimensions. Our models are studied at zero temperature and for finite system sizes. This means there is always a finite gap between the ground and the excited states for a single coboson. In an actual experiment, however, the temperature can never be truly zero, so that thermal effects might mask those purely due to Pauli exclusion. The analysis of thermal states of composite bosons is a delicate task due to the overcompleteness of the coboson basis [7], and lies beyond the scope of the present work.

Our results thus show that the coboson ansatz must be used with caution in situations where its validity has not been tested. We hope that our analysis will spark further interest in the understanding of the very relevant question of when composite particles can be treated as elementary.

\section{ACKNOWLEDGMENTS}

We are grateful to Eloísa Cuestas for discussions on the model and the coboson ansatz. This work used Mendieta Cluster from CCAD-UNC, which is part of SNCAD-MinCyT, Argentina. P.C. acknowledges Consejo Interuniversitario Nacional for the Beca Estímulo a las Vocaciones Científicas, call 


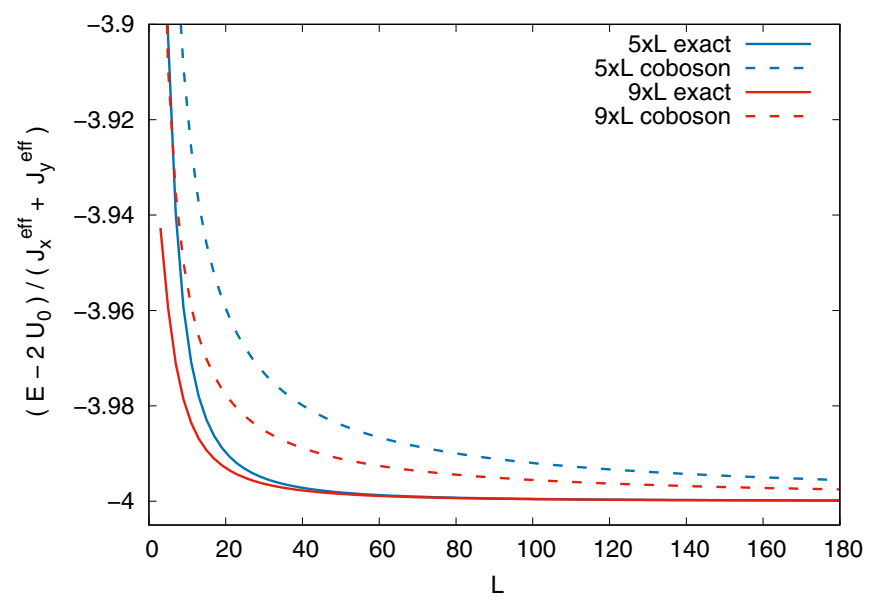

FIG. 6. Exact energy and energy as given by the coboson ansatz for an $n \times L$ lattice with equal tunnelings in both directions, as a function of $L$. All depicted quantities are dimensionless.

2017. P.A.B. gratefully acknowledges support by the Conselho Nacional de Desenvolvimento Científico e Tecnológico do Brasil and by the Spanish MINECO project FIS201459311-P (co-financed by FEDER). C.C. acknowledges funding from Grant No. PICT-BID 2015-2236 from Agencia Nacional de Promoción Científica y Tecnológica.

\section{APPENDIX: ANALYSIS OF THE GROUND-STATE ENERGY FOR TWO-DIMENSIONAL LATTICES}

For completeness, here we analyze the behavior of the energy for the $n \times L$ and $L \times L$ cases discussed in Sec. V. It is important to emphasize that energy is not a good indicator of the ansatz validity, since as it was shown in the main text even in the one-dimensional case in which the ansatz clearly does not work, the exact and approximated energies approach each other when $L$ tends to infinity.

It is relatively simple to calculate the approximate value of the ground-state energy as the expectation value of the effective Hamiltonian (26) over the coboson ansatz (6). For a lattice of $n \times L$ sites and with effective tunneling constants

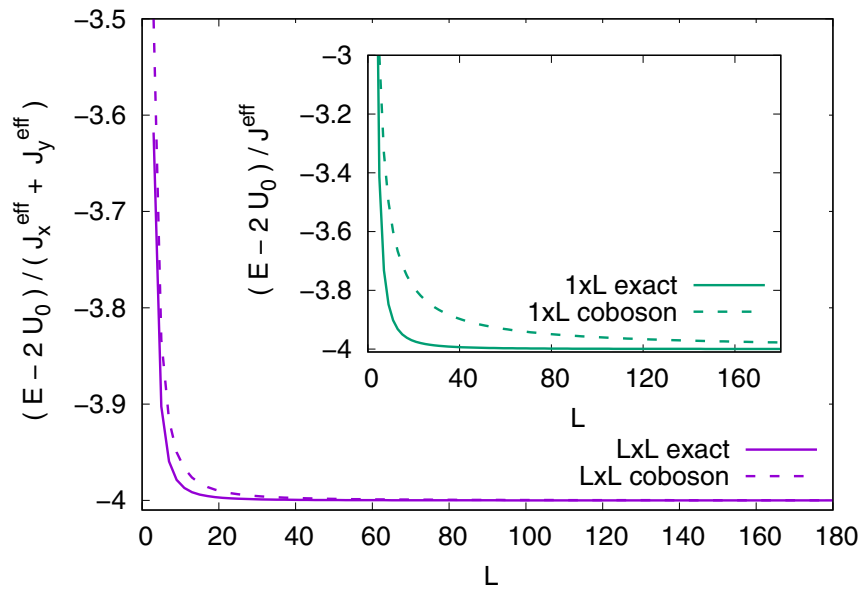

FIG. 7. Exact energy and energy as given by the coboson ansatz for an $L \times L$ lattice with equal tunnelings in both directions, as a function of $L$. Inset: Exact energy and energy as given by the ansatz for a one-dimensional lattice. All depicted quantities are dimensionless.

$J_{v}^{\text {eff }}, v=x, y$, the energy takes the form

$$
E_{G, N=2} \simeq-2 U_{0}-4\left(J_{x}^{\mathrm{eff}}+J_{y}^{\mathrm{eff}}\right)+4\left(J_{x}^{\mathrm{eff}}+J_{y}^{\mathrm{eff}}\right) \frac{1}{L n-1} .
$$

In Fig. 6 we display the behavior of the ground-state energy obtained from numerical diagonalization and that predicted by the coboson ansatz for the ground state, for two different choices of $n$ and as a function of $L$. As in the one-dimensional case, one can see that even though the fidelity between the two states does not approach 1, the two predictions for the energy approach each other as $L$ is increased. This is a consequence of the fact that as the system becomes more dilute, interactions become negligible and the total energy of the ground state of two pairs is well approximated by twice the energy of a single pair. This observation indicates that the asymptotic behavior of the energy is not a good measure of the accuracy of the coboson ansatz. For comparison, Fig. 7 shows the behavior of the energy obtained numerically and from the coboson ansatz for the cases of $L \times L$ and $1 \times L$ lattices.
[1] M. Fierz and W. Pauli, Proc. R. Soc. London Ser. A 173, 211 (1939).

[2] W. Pauli, Phys. Rev. 58, 716 (1940).

[3] J. F. Annett, Superconductivity, Superfluids, and Condensates (Oxford University Press, Oxford, 2005).

[4] C. K. Law, Phys. Rev. A 71, 034306 (2005).

[5] C. Chudzicki, O. Oke, and W. K. Wootters, Phys. Rev. Lett. 104, 070402 (2010).

[6] M. Combescot, O. Betbeder-Matibet, and F. Dubin, Phys. Rep. 463, 215 (2008).

[7] M. Combescot, S.-Y. Shiau, and Y.-C. Chang, Phys. Rev. Lett. 106, 206403 (2011)

[8] M. Combescot and S.-Y. Shiau, Excitons and Cooper Pairs: Two Composite Bosons in Many-Body Physics (Oxford University Press, New York, 2015).
[9] S.-Y. Shiau, M. Combescot, and Y.-C. Chang, Phys. Rev. A 94, 052706 (2016).

[10] M. Combescot and D. W. Snoke, Phys. Rev. B 78, 144303 (2008).

[11] M. Combescot, S.-Y. Shiau, and Y.-C. Chang, Phys. Rev. A 93, 013624 (2016).

[12] P. A. Bouvrie, M. C. Tichy, and I. Roditi, Phys. Rev. A 95, 023617 (2017).

[13] P. A. Bouvrie, E. Cuestas, I. Roditi, and A. P. Majtey, Phys. Rev. A 99, 063601 (2019).

[14] M. C. Tichy, P. A. Bouvrie, and K. Mølmer, Phys. Rev. Lett. 109, 260403 (2012).

[15] P. A. Bouvrie, M. C. Tichy, and K. Mølmer, Phys. Rev. A 94, 053624 (2016). 
[16] S.-Y. Shiau, A. Chenu, and M. Combescot, New J. Phys. 21, 043041 (2019).

[17] Y. H. Pong and C. K. Law, Phys. Rev. A 75, 043613 (2007).

[18] M. Combescot, X. Leyronas, and C. Tanguy, Eur. Phys. J. B 31, 17 (2003).

[19] M. C. Tichy, P. A. Bouvrie, and K. Mølmer, Appl. Phys. B 117, 785 (2014).

[20] I. G. Macdonald, Symmetric Functions and Hall Polynomials (Clarendon Press, Oxford, 1995).

[21] M. Combescot, Europhys. Lett. 96, 60002 (2011).

[22] M. C. Tichy, P. A. Bouvrie, and K. Mølmer, Phys. Rev. A 86, 042317 (2012).
[23] R. J. Baxter, Exactly Solved Models in Statistical Mechanics (Academic Press, London, 1982).

[24] H. Bethe, Z. Phys. 71, 205 (1931).

[25] M. Karabach, G. Muller, H. Gould, and J. Tobochnik, Comput. Phys. 11, 36 (1997).

[26] S. Blundell, Magnetism in Condensed Matter, Oxford Master Series in Condensed Matter Physics (Oxford University Press, Oxford, 2001).

[27] P. Céspedes, Master's thesis, FaMAF, Universidad Nacional de Córdoba, 2018.

[28] Z. Lasmar, P. A. Bouvrie, A. S. Sajna, M. C. Tichy, and P. Kurzynski, arXiv:1902.08157. 